\title{
THE ANGULAR CORRELATIONS OF GALAXIES IN THE COSMOS FIELD ${ }^{1}$
}

\author{
H. J. McCracken, ${ }^{2,3}$ J. A. Peacock, ${ }^{4}$ L. Guzzo,${ }^{5,6,7}$ P. Capak,${ }^{8,9}$ C. Porciani, ${ }^{10}$ N. Scoville,${ }^{8,9}$ H. Aussel, ${ }^{11}$ \\ A. Finoguenov, ${ }^{6}$ J. B. James, ${ }^{4}$ M. G. Kitzbichler, ${ }^{6}$ A. Koekemoer, ${ }^{12}$ A. Leauthaud, ${ }^{13}$ \\ O. Le Fèvre, ${ }^{13}$ R. Massey, ${ }^{8}$ Y. Mellier, ${ }^{2,3}$ B. Mobasher, ${ }^{12}$ P. Norberg, ${ }^{4}$ \\ J. Rhodes, ${ }^{8,14}$ D. B. SAnders, ${ }^{15}$ S. S. SASAKI, ${ }^{16,17}$ Y. Taniguchi, ${ }^{17}$ \\ D. J. Thompson, ${ }^{18,19}$ S. D. M. White, ${ }^{6}$ AND A. El-ZanT ${ }^{20}$ \\ Received 2006 November 10; accepted 2007 April 3
}

\begin{abstract}
We present measurements of the two-point galaxy angular correlation function $w(\theta)$ in the COSMOS field. Independent determinations of $w(\theta)$ as a function of magnitude limit are presented for both the Hubble Space Telescope (HST) ACS catalog and also the ground-based data from Subaru and the Canada-France-Hawaii Telescope. Despite having significantly different masks, these three determinations agree well. At bright magnitudes $\left(I_{\mathrm{AB}}<22\right)$, our data generally match very well with existing measurements and with mock catalogs based on the semianalytic galaxy formation calculations of Kitzbichler \& White (2007) from the Millennium Simulation. The exception is that our result is at the upper end of the expected cosmic variance scatter for $\theta>10^{\prime}$, which we attribute to a particularly rich structure known to exist at $z \simeq 0.8$. For fainter samples, however, the level of clustering is somewhat higher than reported by some previous studies; in all three catalogs we find $w\left(\theta=1^{\prime}\right) \simeq 0.014$ at a median $I_{\mathrm{AB}}$ magnitude of 24 . At these very faintest magnitudes, our measurements agree well with the latest determinations from the Canada-France Legacy Survey. This level of clustering is approximately double what is predicted by the semianalytic catalogs (at all angles). The semianalytic results allow an estimate of cosmic variance, which is too small to account for the discrepancy. We therefore conclude that the mean amplitude of clustering at this level is higher than previously estimated.
\end{abstract}

Subject headings: cosmology: observations — dark matter — galaxies: evolution — galaxies: formation — large-scale structure of universe - surveys

Online material: color figures

\section{INTRODUCTION}

The COSMOS field (Scoville et al. 2007a) is the largest contiguous multiwavelength probe of the high-redshift galaxy distribution, and a major task for the survey will be to extract improved measurements of galaxy clustering at these early times. In this initial paper, we will be concerned with the simplest of these measures, the angular two-point correlation function, $w(\theta)$. Demonstrating a robust measurement of this quantity is a minimum

\footnotetext{
1 Based on observations with the NASA/ESA Hubble Space Telescope, obtained at the Space Telescope Science Institute, which is operated by the Association of Universities for Research in Astronomy (AURA), Inc., under NASA contract NAS 5-26555; also based on data collected at: the Subaru Telescope, which is operated by the National Astronomical Observatory of Japan; the XMM-Newton, an ESA science mission with instruments and contributions directly funded by ESA Member States and NASA; the European Southern Observatory under Large Program 175.A-0839, Chile; Kitt Peak National Observatory, Cerro Tololo Inter-American Observatory, and the National Optical Astronomy Observatory, which are operated by AURA under cooperative agreement with the National Science Foundation; the National Radio Astronomy Observatory, which is a facility of the National Science Foundation, operated under cooperative agreement by Associated Universities, Inc.; and the Canada-France-Hawaii Telescope (CFHT) with MegaPrime/MegaCam, operated as a joint project by the CFHT Corporation, CEA/DAPNIA, the National Research Council of Canada, the Canadian Astronomy Data Centre, the Centre National de la Recherche Scientifique de France, TERAPIX, and the University of Hawaii.

2 Institut d'Astrophysique de Paris, UMR 7095 CNRS, Universitè Pierre et Marie Curie, 98 bis Boulevard Arago, F-75014 Paris, France.

3 Observatoire de Paris, LERMA, 61 Avenue de l'Observatoire, F-75014 Paris, France.

4 Institute for Astronomy, University of Edinburgh, Royal Observatory, Blackford Hill, Edinburgh EH9 3HJ, UK.

5 INAF-Osservatorio Astronomico di Brera, Via Bianchi 46, I-23807 Merate (LC), Italy.

${ }_{6}$ Max-Planck-Institut für Astrophysik, D-85748 Garching bei München, Germany.
}

requirement for verifying that the survey completeness is understood, as a basis for future, more elaborate analysis. The main aim of this paper is therefore to present measurements of the twopoint galaxy clustering statistic on the COSMOS field using three independently generated catalogs, and to compare the results with existing data. We also compare the amplitudes we measure to those found in a semianalytic model of galaxy formation.

The key feature of the COSMOS field is that it is completely covered by the largest existing mosaic of image tiles from the

\footnotetext{
7 Visiting Scientist, European Southern Observatory, Karl-SchwarzschildStrasse 2, D-85748 Garching bei München, Germany.

8 California Institute of Technology, MC 105-24, 1200 East California Boulevard, Pasadena, CA 91125.

${ }^{9}$ Visiting Astronomer, University of Hawaii, 2680 Woodlawn Drive, Honolulu, HI 96822.

${ }^{10}$ Department of Physics, ETH Zurich, CH-8093 Zurich, Switzerland.

11 Service d'Astrophysique, CEA/Saclay, F-91191 Gif-sur-Yvette, France.

12 Space Telescope Science Institute, 3700 San Martin Drive, Baltimore, MD 21218.

${ }^{13}$ Laboratoire d'Astrophysique de Marseille, BP 8, Traverse du Siphon, F-13376 Marseille Cedex 12, France.

14 Jet Propulsion Laboratory, Pasadena, CA 91109.

15 Institute for Astronomy, 2680 Woodlawn Drive, University of Hawaii, Honolulu, HI 96822.

16 Astronomical Institute, Graduate School of Science, Tohoku University, Aramaki, Aoba, Sendai 980-8578, Japan.

17 Physics Department, Graduate School of Science, Ehime University, 2-5 Bunkyo-cho, Matuyama 790-8577, Japan.

18 Large Binocular Telescope Observatory, University of Arizona, 933 North Cherry Avenue, Tucson, AZ 85721-0065.

19 Caltech Optical Observatories, MS 320-47, California Institute of Technology, Pasadena, CA 91125.

20 Canadian Institute for Theoretical Astrophysics, McLennan Labs, University of Toronto, 60 St. George Street, Room 1403, Toronto, ON M5S 3H8, Canada.
} 
Advanced Camera for Surveys (ACS) on the Hubble Space Telescope (HST) (Scoville et al. 2007b). With respect to ground-based surveys, the great advantage of this data set, in addition to the exceptional image quality, is the superior photometric accuracy and stability over the entire field of view of the survey, which in turn makes it possible to measure the clustering of galaxies on large scales and at faint magnitudes where the amplitude of the galaxy correlation function $w(\theta)$ is very small. The COSMOS field is currently the survey that probes the largest comoving scales at redshifts of around 1. The total area covered, $2 \mathrm{deg}^{2}$, is comparable to that of existing studies, in particular the UH8k study $\left(1.5 \mathrm{deg}^{2}\right.$; Wilson 2003) and the shallower DEEP2 measurements (5.0 $\mathrm{deg}^{2}$; Coil et al. 2004). However, COSMOS offers a unique combination of large contiguous area and depth, and also has the virtue of several independent and quite different imaging data sets in the same field.

We use this rich data set to investigate the clustering properties of the field galaxy population on degree scales. In future papers, we will present a more detailed study of galaxy clustering using photometric redshifts which can be used, for example, to divide our galaxy catalogs by type and apparent magnitude. Our objective here is simply to present the global properties of the field in terms of simple two-point statistics for catalogs selected by apparent magnitude. As our aim is to demonstrate the robustness of the results, we restrict ourselves to the $i$-band data, which are available for all three data sets considered here.

The COSMOS field has been imaged by many groundbased facilities, including the Subaru telescope (Taniguchi et al. 2007), and we compare the COSMOS ACS catalog (described in Leauthaud et al. 2007) with two ground-based catalogs: the Subaru optical catalog described by Capak et al. (2007) and the CanadaFrance-Hawaii Telescope Legacy Survey (CFHTLS) T03 catalog used by H. J. McCracken et al. (2007, in preparation). Each of these have significantly different masks, especially with regard to ghosting around bright stars. As will be demonstrated, the results of these independent determinations are in good agreement, and display a consistently higher amplitude at faint magnitudes than has been suggested in previous work.

\section{CATALOGS AND METHODS}

All three of the catalogs were prepared independently. For full details of the ACS catalog, see Leauthaud et al. (2007). The Subaru catalogs are described in Capak et al. (2007). A full description of the CFHTLS catalogs, based on the images corresponding to the release CFHTLS-T03, can be found in H. J. McCracken et al. (2007, in preparation).

The ACS data are constructed from a mosaic of 575 image tiles taken over 588 orbits of the $H S T$. The $50 \%$ completeness limit of the catalog is $26.6 \mathrm{~F} 814 \mathrm{~W}$ magnitudes.

SuprimeCam consists of 10 Lincoln-Labs $8 \mathrm{~K} \times 4 \mathrm{~K}$ CCDs with a plate scale of $0.2^{\prime \prime} \mathrm{pixel}^{-1}$. However, a plate scale of $0.15^{\prime \prime} \mathrm{pixel}^{-1}$ was used for the final image to ensure that the images with good seeing were not undersampled. A special dither pattern including camera rotations (Subaru is an alt-azimuth telescope) was used to ensure that every portion of the field was imaged by at least four different CCDs. Further details can be found in Taniguchi et al. (2007). The Subaru catalog was based on a mosaic of 115 Subaru images taken with the Suprime camera on the Subaru $8 m$ telescope. The image has a median seeing of $0.6^{\prime \prime}$ and a $50 \%$ completeness of $i=27.4$.

In order to produce the best possible catalog for correlation function measurements, we re-extracted a catalog ourselves from the Subaru tiles produced by Capak et al. We downloaded each tile and assembled them into a single large mosaic using the TERAPIX software tool SWarp; we carried out the same proce-

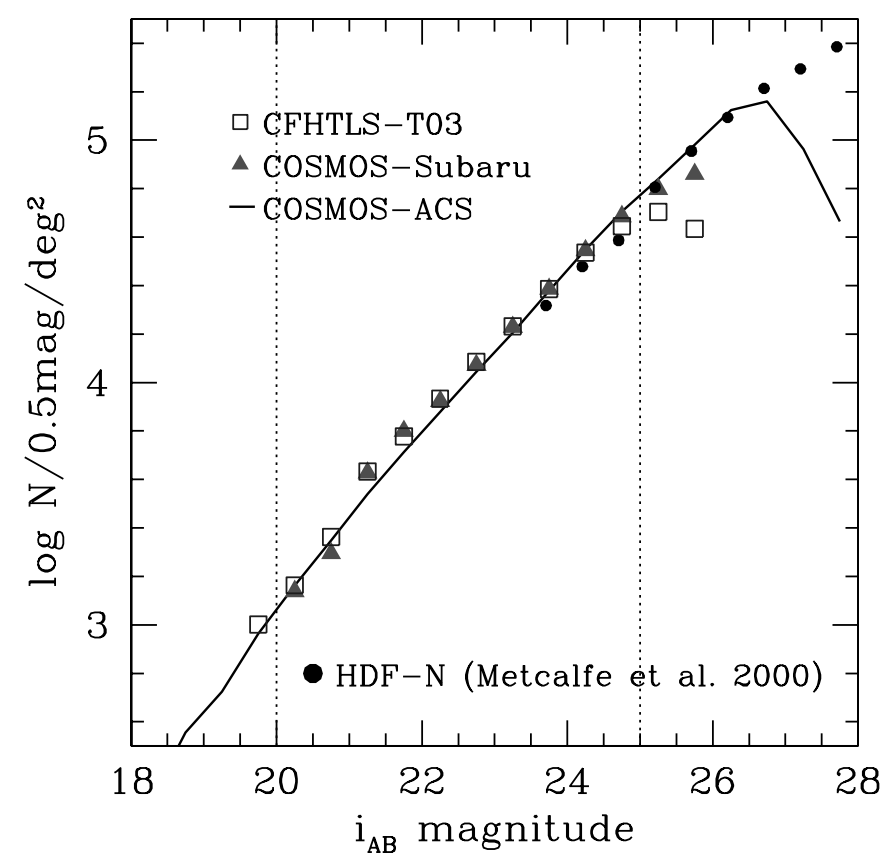

FIG. 1.-Galaxy counts for the three catalogs presented in this paper: ACS (solid line), CFHTLS-T03 (open squares), and Subaru ( filled triangles). For reference, we also show galaxy counts extracted from the HDF-N by Metcalfe et al. (2001). [See the electronic edition of the Supplement for a color version of this figure.]

dure for the rms maps. Following this, we used SExtractor to extract a catalog. Star-galaxy separation to $i=21.5$ was performed using the flux_radius compactness parameter, which measures the radius which encloses $50 \%$ of an object's flux. Bright stars and defects were masked on the images.

The CFHTLS catalog is derived from the TERAPIX CFHTLST03 release. The CFHTLS stacks were taken using the MegaCam camera on the $3.6 \mathrm{~m}$ CFHT telescope. MegaCam covers $1 \mathrm{deg}^{2}$ with $0.205^{\prime \prime}$ pixels using 36 separate $2048 \times 4096$ Rockwell CCDs. Note that, unlike the Subaru and ACS data, the CFHTLS-T03 image consists of a single MegaCam pointing. The CFHTLS-T03 D2-i data set comprises 153 images and has a median seeing of $0.9^{\prime \prime}$. The $50 \%$ completeness of this data set is $I_{\mathrm{AB}} \simeq 25.7 \mathrm{mag} .{ }^{21}$

In all three catalogs, the star-galaxy separation is carried out using a morphological classifier. We emphasize that the classifiers were determined independently for each catalog; we did not, for example, use the ACS morphologies to perform star-galaxy separation on the CFHTLS or Subaru images, or use the Subaru images as detection images for the ACS data. The Subaru images cover the full $2 \mathrm{deg}^{2}$ of the COSMOS field, while the ACS tiling covers a total of $1.7 \mathrm{deg}^{2}$; the CFHTLS-T03 images cover just the central $1 \mathrm{deg}^{2}$ of the field.

In each catalog, regions around bright stars and near the edges of the field were masked. For the ACS catalogs, we used the same set of masks that were used for weak-lensing measurements (Massey et al. 2007). These masks also remove many blended objects. After masking, in the Subaru catalog there are 134,397 galaxies in the magnitude range $20<i<25$; in the ACS catalog there are 124,665 galaxies in the same interval in magnitude. For the CFHTLS, there are 52,521 galaxies in the magnitude range $20<i<24$. The effective areas (total available area after masking) of the three surveys (Subaru, ACS, and CFHT) are 1.6, 1.5, and $0.7 \mathrm{deg}^{2}$, respectively (i.e., completenesses of $80 \%, 88 \%$, and $70 \%$ ). We have experimented with varying the degree of masking

${ }^{21}$ For details see http://terapix.iap.fr/cplt/tab_t03ym.html. 


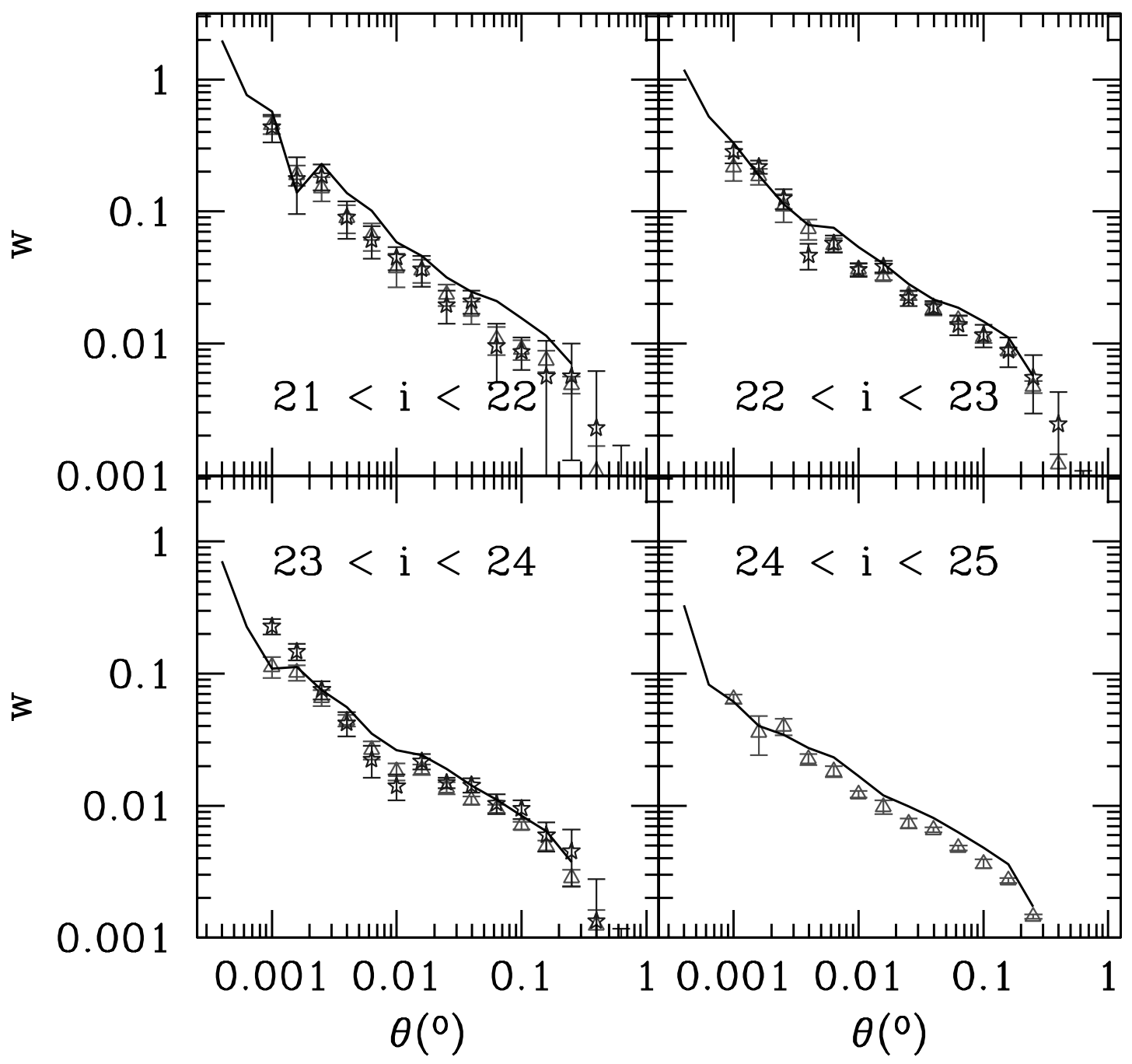

FIG. 2.-Angular correlation function, $w(\theta)$, as a function of angular separation, $\theta$, in four slices of apparent magnitude. In each panel we show three different measurements

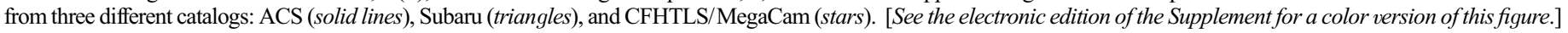

by "growing" the mask to eliminate pixels adjacent to masked pixels. The results are robust even when more than $50 \%$ of the area is masked.

Figure 1 shows the galaxy number counts extracted from the three catalogs. The dotted lines indicate the magnitude limits adopted in this paper. The slight "break" in the counts at $i_{*} \simeq 21$ is an artifact caused by our morphologically based star-galaxy separation. The ACS count is slightly lower at $i \simeq 22$ as a consequence of the improved star-galaxy separation in this data set.

\section{CLUSTERING MEASUREMENTS}

We selected galaxies in progressively fainter slices of apparent $i$ magnitude. For the purposes of the paper we assume that the instrumental AB total magnitudes measured in each catalog are equivalent; this is approximately true. Capak et al. (2007) present a detailed comparison of galaxy photometry between the three catalogs described here. Figure 8 of their paper demonstrates that total instrumental magnitudes in each catalog agree well, to within 0.05 mag.

For each slice, we measure $w$ for a range of angular separations $\theta$ to $\theta+\delta \theta$ in a series of logarithmically separated bins using the standard Landy \& Szalay (1993) estimator,

$$
w(\theta)=\frac{\mathrm{DD}-2 \mathrm{DR}+\mathrm{RR}}{\mathrm{RR}},
$$

with the DD, DR, and RR terms referring to the number of datadata, data-random, and random-random galaxy pairs between $\theta$ and $\theta+\delta \theta$. The fitted amplitudes quoted in this paper assume a power-law slope for the galaxy correlation function, $w(\theta)=$ $A_{w}(\theta / \mathrm{deg})^{-\delta}$; however, this amplitude must be adjusted for the "integral constraint" correction, arising from the need to estimate the mean galaxy density from the sample itself. This can be estimated as (e.g., in Adelberger et al. 2005),

$$
C=\frac{1}{\Omega^{2}} \iint w(\theta) d \Omega_{1} d \Omega_{2},
$$

where $\Omega$ is the area subtended by each of our survey fields. For the COSMOS field, We find $C \sim 1 A_{w}$ by numerical integration of equation (2) over our field geometry and assuming that galaxies closer than $1^{\prime \prime}$ cannot be distinguished.

We used a sorted linked list in order to reduce computing time given the very large number of objects in each slice. These results are compared in Figure 2. The solid lines show measurements from the ACS; the triangles and stars correspond to measurements from Subaru and CFHT. At each angular bin for each survey, the error bars plotted are simple bootstrap errors. Although these are not in general a perfect substitute for a full estimate of cosmic variance (e.g., using an ensemble of simulations), they should give the 


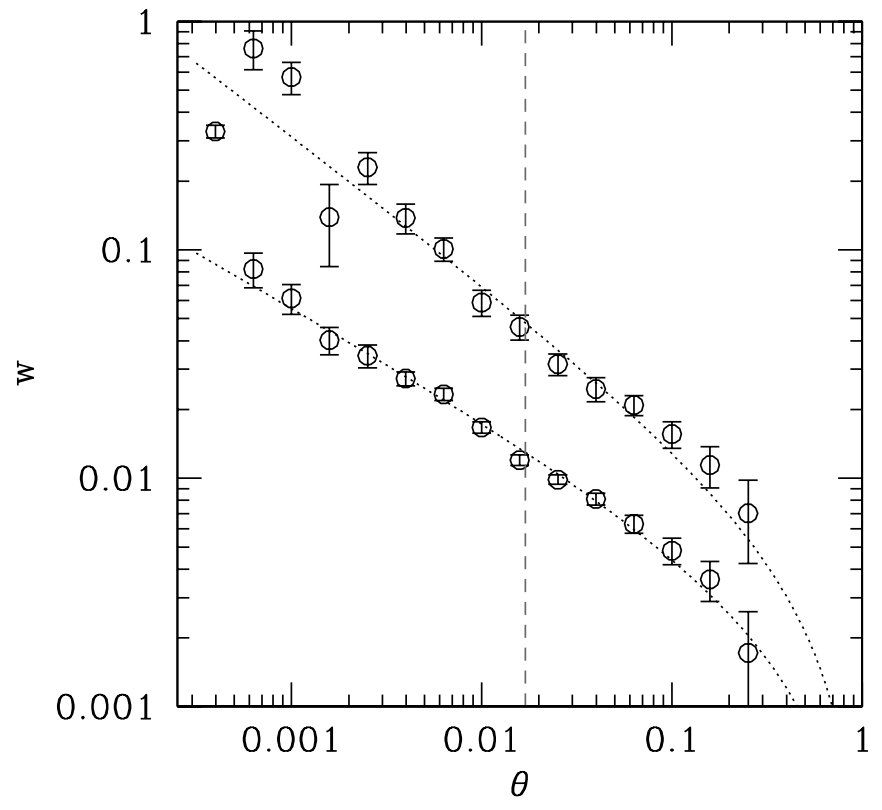

FIG. 3.-Amplitude of the angular correlation function $w$, as a function of angular separation, $\theta$, for the ACS catalog. Measurements for galaxies selected in the magnitude ranges $21<i<22$ and $24<i<25$ are presented. The dotted lines show the best-fitting power-law correlations (with slopes -0.59 and -0.47 , respectively) with the integral correction for the COSMOS field included. The dashed line is plotted at $1^{\prime}$. There seems little evidence here for any deviation from power-law correlations, although the brighter bin, represented by the upper line, does hint at an inflection around $1^{\prime}$. The correlation function for the fainter bin is clearly flatter. [See the electronic edition of the Supplement for a color version of this figure.]

correct magnitude of the uncertainty (Mo et al. 1992). In particular, Mo et al. show that bootstrap errors yield sensible uncertainties on power-law fits to correlation-function data when the points are treated as independent.

For each of the four slices in apparent magnitude, the amplitude of $w(\theta)$ measured in the Subaru data agrees well with the measurements in the ACS. At very small angular separations the ACS data are higher than the ground-based results. For the two faintest bins (23 $<i<24$ and $24<i<25$ ), the agreement between the ACS measurements and the Subaru measurements is excellent.

We now examine more closely the galaxy correlation function measured from the ACS catalogs. As we have already seen, thanks to the excellent resolution of the ACS images, we are able to measure clustering amplitudes to small separations, on the order of $1^{\prime \prime}$. Conversely, as a consequence of the large areal coverage of the ACS COSMOS field, we can also measure amplitudes to large angular separations. In Figure 3, we show the angular correlation function $w(\theta)$ as a function of angular separation for bright and faint samples with $21<i<22$ and $24<i<25$, respectively. The dotted lines show the best-fitting lines with an integral constraint correction applied. For the bright bin, we find a best-fitting slope of $-0.59 \pm 0.05$; for the fainter bin, $-0.47 \pm 0.02$. We find unambiguously that in this magnitude-limited sample, the galaxy correlation function becomes flatter toward fainter magnitude bins, in agreement with previous works (McCracken et al. 2001).

\section{COMPARISONS WITH SIMULATIONS}

In the previous sections, we established that measurements between the different data sets are consistent. In this section, we compare our measurements to those made on catalogs produced by Kitzbichler \& White (2007). These catalogs were created using a semianalytic model to simulate galaxy formation within the evolving halo population of the extremely large Millennium Sim- ulation (MS). These simulated universes were then "observed" to produce light cones, which can then be used to produce observations with geometry identical to those of real catalogs. (Blaizot et al. 2005; Kitzbichler \& White 2007). Each COSMOS light cone covers $2 \mathrm{deg}^{2}$. Figure 4 shows, as before, the amplitude of $w(\theta)$ as a function of angular separation in four magnitude slices. Points with error bars show measurements from the ACS data. The solid line shows the average of measurements made from 20 light cones extracted from the MS; the dotted lines show the amplitude of the $\pm 1 \sigma$ error bars.

For the two brighter slices, $21<i<22$ and $22<i<23$, the agreement between the simulations and observations at intermediate and small scales is remarkably good. At fainter magnitudes, and at larger scales, the amplitudes measured in the COSMOS catalogs are consistently higher than the prediction of the simulations. Since the simulations allow us to assess the amplitude of cosmic variance directly, we can easily conclude that this discrepancy cannot plausibly be attributed to the COSMOS field having above-average clustering. It therefore appears that the model predictions for $w(\theta)$ at $i \simeq 24$ are too low; we discuss this further below.

\section{COMPARISONS \\ WITH LITERATURE MEASUREMENTS}

From our measurements, it is clear that the form of $w(\theta)$ in the COSMOS field does not correspond to a simple power law with a slope that is independent of the median magnitude of the sample. In the past, determinations of galaxy clustering amplitude were usually given at a fixed angular scale over a small range of angular separations. From our data, it is clear that fitting $w(\theta)$ only at small angular separations will result in different fitted amplitudes as compared to a fit over the entire range. Therefore, in order to compare with results presented in the literature, we choose to carry out fits over a similar range of angular separations.

In Figure 5, we show the fitted amplitudes of $w(\theta)$ as a function of the median magnitude of each slice. In comparing with literature measurements, we see that at bright magnitudes $(i \simeq 20)$ our measurements are approximately in agreement with the values presented in the literature. However, by $i \simeq 23$, the COSMOS field measurements are significantly higher than, for example, the Canada-France Deep Fields measurements of McCracken et al. (2001). This is true at all angles and for all measurements, whether they are from the ground-based or space-based catalogs.

Photometric redshifts are available for all objects in the COSMOS field (Mobasher et al. 2007). The slice with $22<i<$ 23 has a median redshift of $z \simeq 0.8$; the next faintest slice is at $z \simeq 0.9$. If we examine Figure 2 of Scoville et al. (2007c), we can see there is a significant overdensity in the redshift range $0.7<$ $z<0.9$. It is possible that this structure could contribute to the enhanced signal on large scales seen in our data in these slices.

Some independent evidence exists that this structure raises the COSMOS clustering amplitude above the ensemble average. In Figure 5, we have plotted the average amplitude of $w$ as a function of apparent median magnitude for the four independent deep fields of the Canada-France-Hawaii Telescope Legacy Survey (one of which is the D2 field described above), totaling an effective area of $3.2 \mathrm{deg}^{2}$. Error bars correspond to the variance over all four fields. Interestingly, at bright magnitudes, the CFHTLS magnitudes agree with the COSMOS measurements and other literature values; at intermediate magnitudes $(22<i<23)$, they are between the COSMOS values and those from other surveys, whereas at fainter magnitudes they agree perfectly with the COSMOS measurements, presumably because the median redshift probed by both surveys at these magnitude limits is beyond that of the rich structure in 


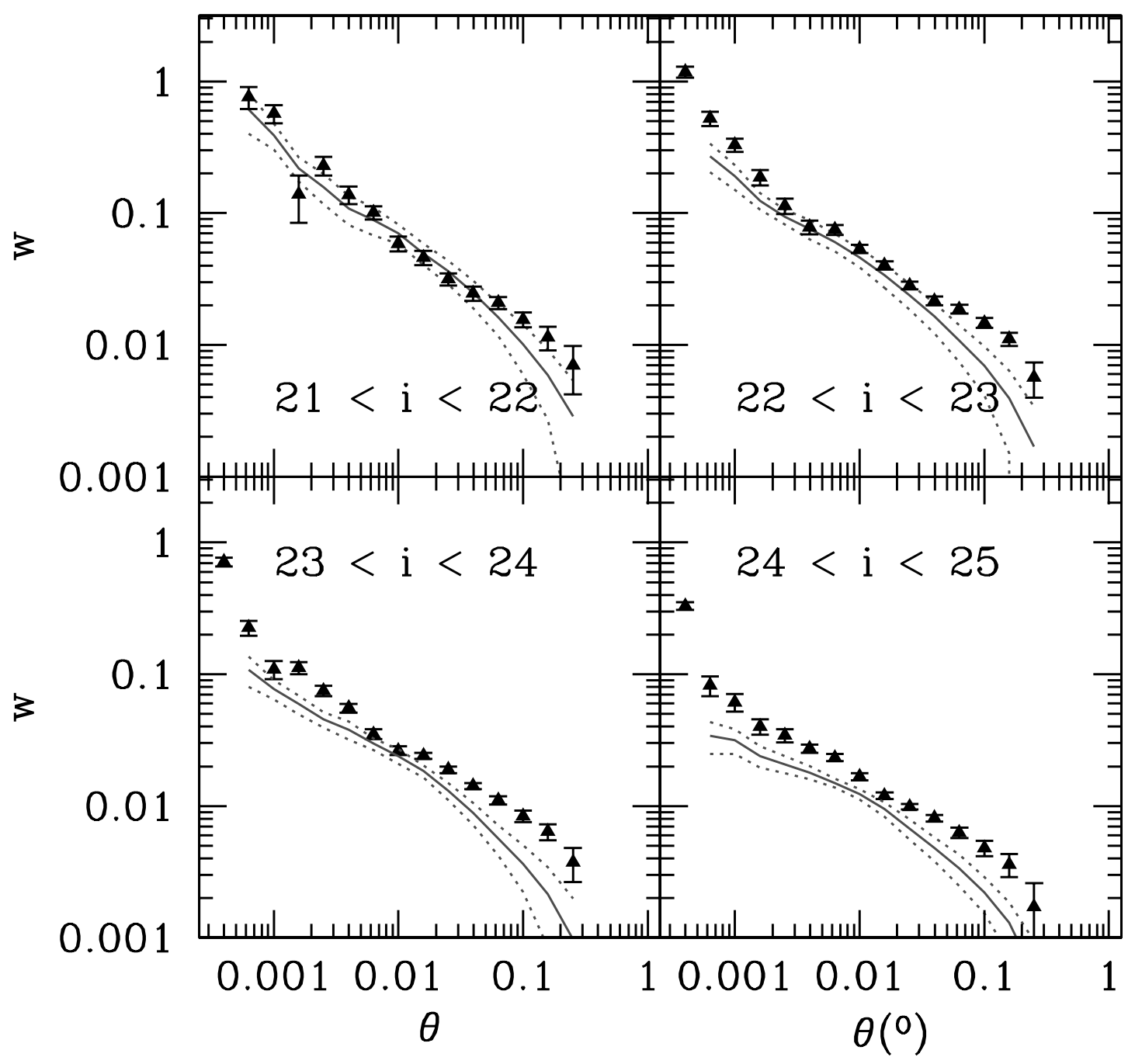

FIG. 4.-Amplitude of the angular correlation function $w$, as a function of angular separation, $\theta$, for the ACS catalog. The solid lines show measurements made using mock catalogs extracted from the MS. The dotted lines show the $1 \sigma$ scatter of the results for individual mock catalogs around their mean, computed from the variance over twenty mock catalogs. Note that these mock results automatically incorporate integral corrections due to the finite field size. [See the electronic edition of the Supplement for a color version of this figure.] 


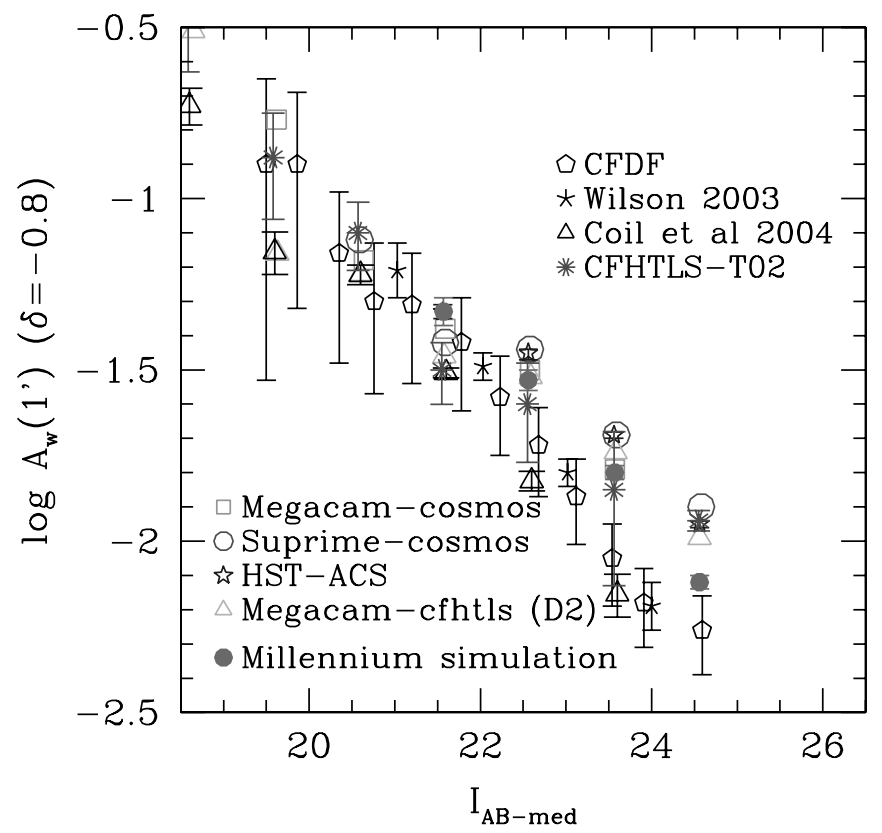

FIG. 5.-Comparisons of the COSMOS results for the dependence of the amplitude of $w(\theta)$ on depth, compared with existing measurements and with the mock results. We choose to specify the amplitude at $1^{\prime}$, based on a $\delta=-0.8$ power-law fit around this point. The results are highly insensitive to this choice of slope. [See the electronic edition of the Supplement for a color version of this figure.]

the COSMOS field. The size of the cosmic variance error bars on the CFHTLS measurements is also largest at intermediate magnitudes. These measurements are discussed in greater detail in a forthcoming paper (H. J. McCracken et al., in preparation).

\section{DISCUSSION AND CONCLUSIONS}

This paper has presented measurements of the angular twopoint correlation function in the COSMOS field, $w(\theta)$, and how it depends on $i$-band magnitude depth. We have shown that consistent results are obtained using three independent data sets: HST ACS, Subaru SuprimeCam, and CFHT MegaCam. The results agree well at bright magnitudes $(i \simeq 22)$ with previous measurements and with the predictions of semianalytic mock catalogs constructed from the MS. The only slight caveat here is that the results at $\theta>10^{\prime}$ are at the high end of the MS predictions, which may reflect a single rich $z \simeq 0.8$ structure in the field.

At fainter magnitudes, however, a different picture emerges. By the time we reach the $24<i<25$ bin, the COSMOS measurements are consistently a factor 2 higher than the MS predictions at all angles. Moreover, the COSMOS measurements are consistent with the four-field average of measurements from the CFHTLS survey. This discrepancy is well beyond the compass of cosmic variance from limited numbers of rich structures, as measured via the ensemble of simulations. Thus, barring some undetected systematic that is consistent between all the data sets we have used, the conclusion must be that the MS predictions are too low at these magnitude levels. This could arise in a number of ways: the predicted degree of bias at high redshifts might be too low; the luminosity function might be incorrect, resulting in too high a predicted mean redshift at these depths; or, alternatively, the MS may miss foreground pairs of intrinsically faint galaxies because of the resolution limit of the simulation. The first possibility is particularly interesting given the current debate over the normalization of the primordial power spectrum, $\sigma_{8}$. The MS used $\sigma_{8}=0.9$, whereas $W M A P$ favors a smaller result, perhaps as low as $\sigma_{8}=0.7$ (Spergel et al. 2007). Since high-redshift galaxies are strongly biased already, a reduced $\sigma_{8}$ will in fact boost the predicted galaxy clustering (for a given galaxy mass). These issues will be explored further in future papers, where we make direct use of the photometric redshift data in the COSMOS field.

The HST COSMOS Treasury program was supported through NASA grant HST-GO-09822. We wish to thank Tony Roman, Denise Taylor, and David Soderblom for their assistance in planning and scheduling the extensive COSMOS observations. We gratefully acknowledge the contributions of the entire COSMOS collaboration, consisting of more than 70 scientists. More information on the COSMOS survey is available at http://www.astro .caltech.edu/ cosmos. It is a pleasure to acknowledge the excellent services provided by the NASA IPAC/IRSA staff (Anastasia Laity, Anastasia Alexov, Bruce Berriman, and John Good) in providing online archive and server capabilities for the COSMOS data sets.

\section{REFERENCES}

Adelberger, K. L., Steidel, C. C., Pettini, M., Shapley, A. E., Reddy, N. A., \& Erb, D. K. 2005, ApJ, 619, 697

Blaizot, J., Wadadekar, Y., Guiderdoni, B., Colombi, S. T., Bertin, E., Bouchet, F. R., Devriendt, J. E. G., \& Hatton, S. 2005, MNRAS, 360, 159 Capak, P. L., et al. 2007, ApJS, 172, 99

Coil, A. L., Newman, J. A., Kaiser, N., Davis, M., Ma, C.-P., Kocevski, D. D., \& Koo, D. C. 2004, ApJ, 617, 765

Kitzbichler, M. G., \& White, S. D. M. 2007, MNRAS, 376, 2

Landy, S. D., \& Szalay, A. S. 1993, ApJ, 412, 64

Leauthaud, A., et al. 2007, ApJS, 172, 219

Massey, R., et al. 2007, ApJS, 172, 239

McCracken, H. J., Le Fèvre, O., Brodwin, M., Foucaud, S., Lilly, S. J., Crampton, D., \& Mellier, Y. 2001, A\&A, 376, 756

Metcalfe, N., Shanks, T., Campos, A., McCracken, H. J., \& Fong, R. 2001, MNRAS, 323, 795

Mo, H. J., Jing, Y. P., \& Boerner, G. 1992, ApJ, 392, 452

Mobasher, B., et al. 2007, ApJS, 172, 117

Scoville, N. Z., et al. 2007a, ApJS, 172, 1

2007b, ApJS, 172, 38

. 2007c, ApJS, 172, 150

Spergel et al. 2007, ApJS, 170, 377

Taniguchi, Y, et al. 2007, ApJS, 172, 9

Wilson, G. 2003, ApJ, 585, 191 